\title{
Community-acquired Escherichia coli meningitis with ventriculitis in an adult-a rare case report
}

\author{
Rajesh Kasimahanti ${ }^{{ }^{*}}$ (D) Sai Kandraju Satish ${ }^{2}$ and Mridu Anand $^{3}$
}

\begin{abstract}
Background: Community-acquired gram-negative bacillary meningitis is rare to occur without preexisting conditions like trauma, organ dysfunction, and immunocompromised state, and very few case reports with Escherichia coli have been described in literature till now. Presence of ventriculitis along with meningitis makes the incidence further sparse.

Case presentation: A review of literature identified a total of only 45 community-acquired E. coli meningitis from 1945 till to date. Here, we have described a case of community-acquired $E$. coli meningitis with ventriculitis in an adult with past history of completely repaired CSF leak secondary to trauma nearly 23 years ago, without current radiological evidence of persistent CSF leak and therefore described as spontaneously acquired. Post-contrast T1 images of MRI were suggestive of subtle ependymal enhancement of ventricles, and patient was treated in lines of ventriculitis. Initial CSF was suggestive of acute pyogenic meningitis, and the organism grown was pan-sensitive E. coli. Patient was treated with antibiotics according to the culture sensitivity pattern and was given a prolonged course of 6 weeks of antibiotic therapy in view of ventriculitis.
\end{abstract}

Conclusion: Community-acquired E. coli meningitis with possible ventriculitis in adults is described as a rare entity and is likely to be underrated and under-recognized.

Keywords: Community-acquired meningitis, Ventriculitis, E. coli, CSF leak

\section{Background}

Gram-negative bacilli (GNB) are a relatively uncommon cause of community-acquired meningitis in adults ranging from 0.7 to $7 \%$ across the world [1]. Most of the GNB meningitis occurs after a neurosurgical procedure, within a month of head trauma, in the presence of a neurosurgical device or any cerebrospinal fluid (CSF) leak syndrome. They represent $75 \%$ of the cases of nosocomial gram-negative bacillary meningitis [2]. The reported annual incidence of spontaneous GNB meningitis in adults is around 2 cases per 100,000 adults. The highest reported incidence is around $8.7 \%$, of which, Escherichia coli (E. coli) represents around 41.9\% [3]. Post-traumatic CSF leak predisposing GNB meningitis usually presents within a month of trauma, and the risk reduces after the repair of leak without any residual defect. At the same

\footnotetext{
* Correspondence: rajeshkasimahanti@gmail.com

'Department of Critical Care Medicine, Yashoda Hospitals, Alexander Road,

PIN: 500003, Secunderabad, Telangana, India

Full list of author information is available at the end of the article
}

time, presence of ventriculitis along with community-acquired meningitis is much rare. To the best of our knowledge, there were no case reports hitherto of E. coli meningitis with ventriculitis. Here, we are describing a case of community-acquired spontaneous $E$. coli meningitis with ventriculitis which presented 23 years after complete repair of CSF leak for head trauma.

\section{Case presentation}

A 56-year-old male patient with no known comorbidities presented to the emergency department with history of fever and headache for past 2 days with sporadic episodes of projectile vomiting, irrelevant talk, and poor sensorium for a day. On admission, the patient manifested fever $\left(39^{\circ} \mathrm{C}\right)$, a heart rate of 104 beats per minute, blood pressure of $130 / 60 \mathrm{mmHg}$, respiratory rate of $30 / \mathrm{min}$, and capillary blood glucose of $140 \mathrm{mg} / \mathrm{dl}$. His Glasgow Coma Scale (GCS) score was 9, with respective scores of 5, 2, and 2 for motor, eye, and verbal responses. There were no signs of meningeal irritation or focal 
neurological deficit. Clinical examination was otherwise normal except for a scar over the anterior bi-frontal region which was a surgical incision. On further inquiry, he had an accidental fall from a two-story building 23 years ago, causing a persistent CSF leak from the nose (rhinorrhea). He underwent complete repair for the CSF leak without any residual defect. There were no further hospitalizations for the past 23 years with symptoms suggestive of meningitis.

His computerized tomography (CT) scan showed cystic gliosis changes in the left frontal lobe which was communicating with the frontal horn of ipsilateral lateral ventricle. CSF analysis of the patient revealed hypoglycorrachia $(<20 \mathrm{mg} / \mathrm{dl}$ with corresponding blood sugar of $140 \mathrm{mg} / \mathrm{dl}$ ), elevated protein $>300 \mathrm{mg} / \mathrm{dl}$, and neutrophil-predominant pleocytosis (total cells $360 / \mathrm{mm}^{3}$ with $96 \%$ polymorphs) suggestive of acute pyogenic meningitis. He was started empirically on ceftriaxone $100 \mathrm{mg} / \mathrm{kg} /$ day in two divided doses, amoxycillin $100 \mathrm{mg} / \mathrm{kg} /$ day in four divided doses, and acyclovir $45 \mathrm{mg} / \mathrm{kg} /$ day in three divided doses. Initial peripheral blood WBC count was 14,760 with $85 \%$ neutrophils and serum lactate levels of $5.35 \mathrm{mmol} / \mathrm{l}$. His renal and liver function tests were normal. Antibiotics (ceftriaxone, amoxycillin) were continued, and acyclovir was stopped as there were filamentous gram-negative rods in gram staining of the CSF, which was a rare morphology to be identified (Fig. 1). Further, the sample was plated onto blood agar, MacConkey agar, and chocolate agar and incubated aerobically at $37^{\circ} \mathrm{C}$. Non-hemolytic colonies were formed on blood agar (Fig. 2) which were lactose-fermenting colonies on MacConkey agar (Fig. 3). The organism was identified to be Escherichia coli by Vitek 2 Compact (Biomerieux), and spectrum of antibiotic sensitivity was described in Table 1.

\section{Further course}

In accordance with the culture and sensitivity pattern, ceftriaxone was continued and amoxycillin was stopped.

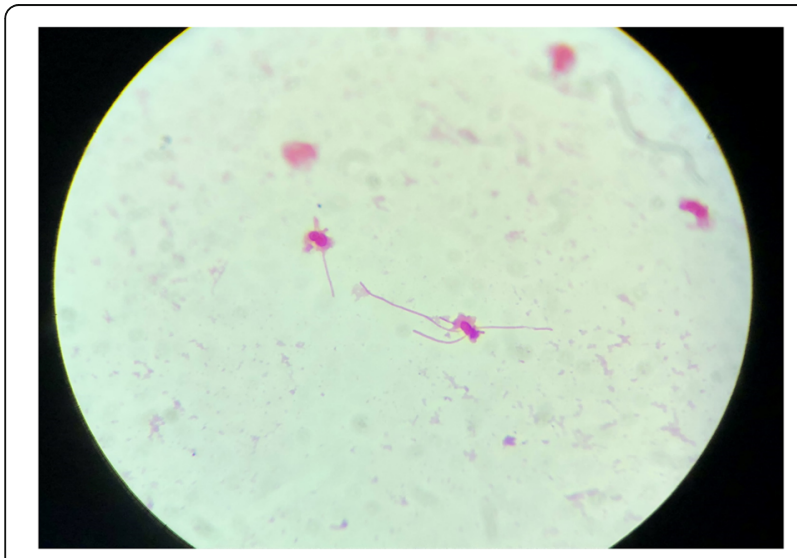

Fig. 1 Gram-negative filamentous rods

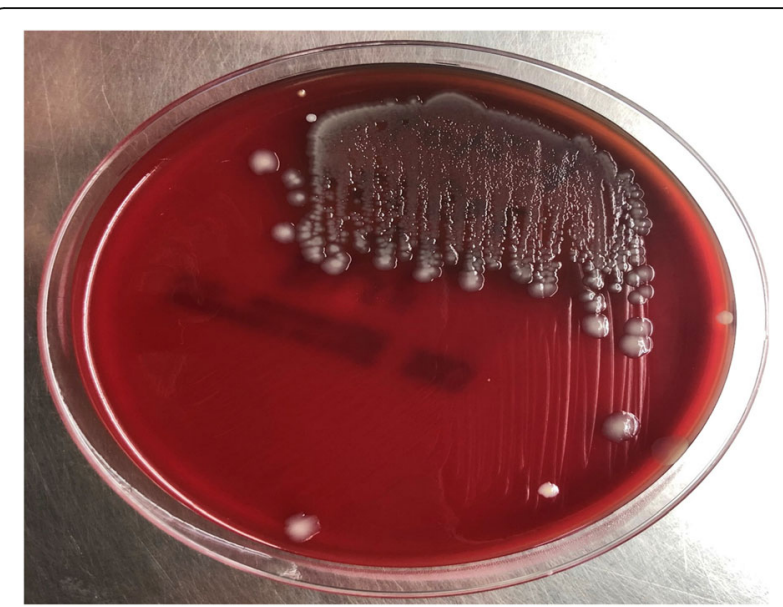

Fig. 2 Non-hemolytic colonies on blood agar

He was afebrile for the next $48 \mathrm{~h}$, and his sensorium improved. Further work up was done to identify the source of seeding of $E$. coli to the meninges. Urine culture and blood cultures that were sent prior to initiation of antibiotics did not show any bacterial growth, and stool microscopy was negative for ova and cysts. CT scan of the abdomen revealed a non-obstructive renal calculus of $6 \mathrm{~mm} \times 6 \mathrm{~mm}$ at interpole of the left kidney without any signs of pyelonephritis. After the initial improvement, patient again had a drop in sensorium without any localizing signs. Magnetic resonance imaging (MRI) of the brain with contrast study was done to rule out secondary complications associated with meningitis like vascular infarcts or hydrocephalus. There was post-contrast enhancement of leptomeninges and subtle enhancement of ventricular wall and posterior fossa cisternal spaces in post-contrast $\mathrm{T} 1$ sequence, and the same findings along with exudates in the ventricles in T2 fluid attenuated inversion recovery (FLAIR) images, which were suggestive

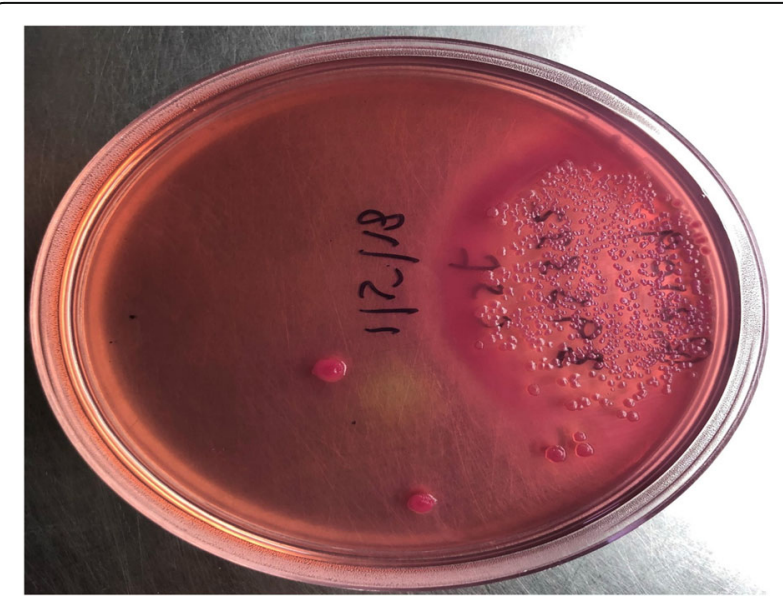

Fig. 3 Lactose-fermenting colonies on MacConkey agar 
Table 1 Spectrum of Antimicrobial sensitivity

\begin{tabular}{lll}
\hline Organism: Escherichia coli & & \\
\hline Antibiotic & MIC & Sensitivity \\
\hline Ampicillin & $>32$ & $\mathrm{R}$ \\
Cefuroxime & $=4$ & $\mathrm{~S}$ \\
Ceftriaxone & $<1$ & $\mathrm{~S}$ \\
Cefipime & $<1$ & $\mathrm{~S}$ \\
Meropenem & $<0.25$ & $\mathrm{~S}$ \\
\hline
\end{tabular}

of ventriculitis (Figs. 4 and 5). CT cisternogram was performed to check for residual CSF leak at the past surgical site, in which there was an opacified density in the left frontal region representing a porencephalic cyst communicating with the left frontal horn. There was no CSF leak into the paranasal sinuses or nasal cavity (Fig. 6).

In the CSF analysis repeated on day 7 of antibiotic therapy, there was improvement in cell count (total cell count of 26 with $100 \%$ of lymphocytes) and cultures were sterile. He was discharged from the hospital on day 10 and advised to continue intravenous antibiotics for 6 weeks in view of ventriculitis. On further follow-up, the patient has improved, with no neurological deficit and antibiotics have been stopped at the end of the sixth week.

\section{Discussion and conclusions}

Community-acquired spontaneously occurring E. coli meningitis in adults is rare, and the recent report by Bichon et al. showed a total of 45 cases of the same from 1945 to 2017 with an average report of one case per year

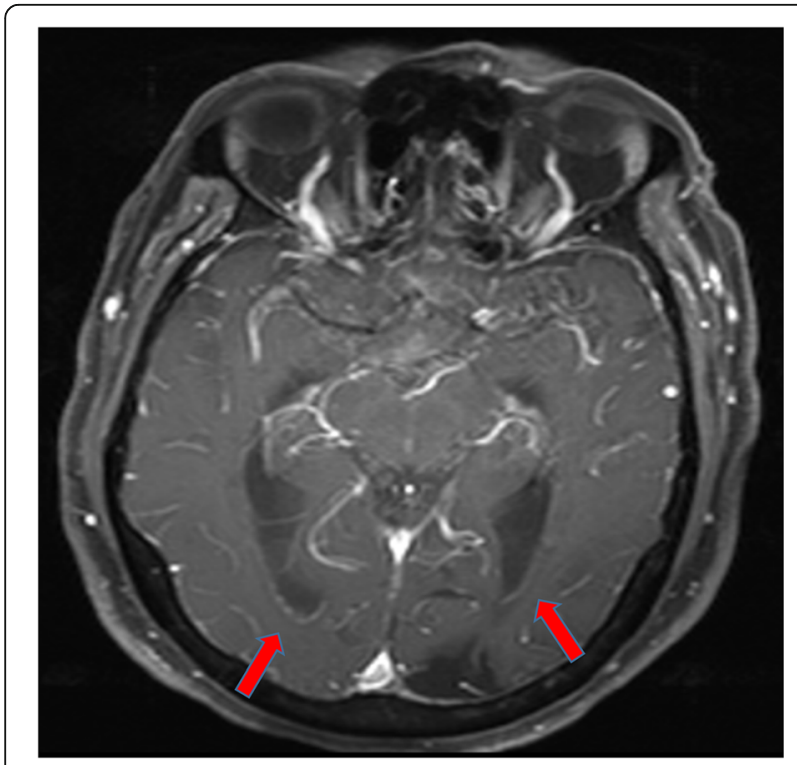

Fig. 4 Post-gadolinium T1 image showing subtle ventricular ependymal enhancement (arrow) in occipital horns

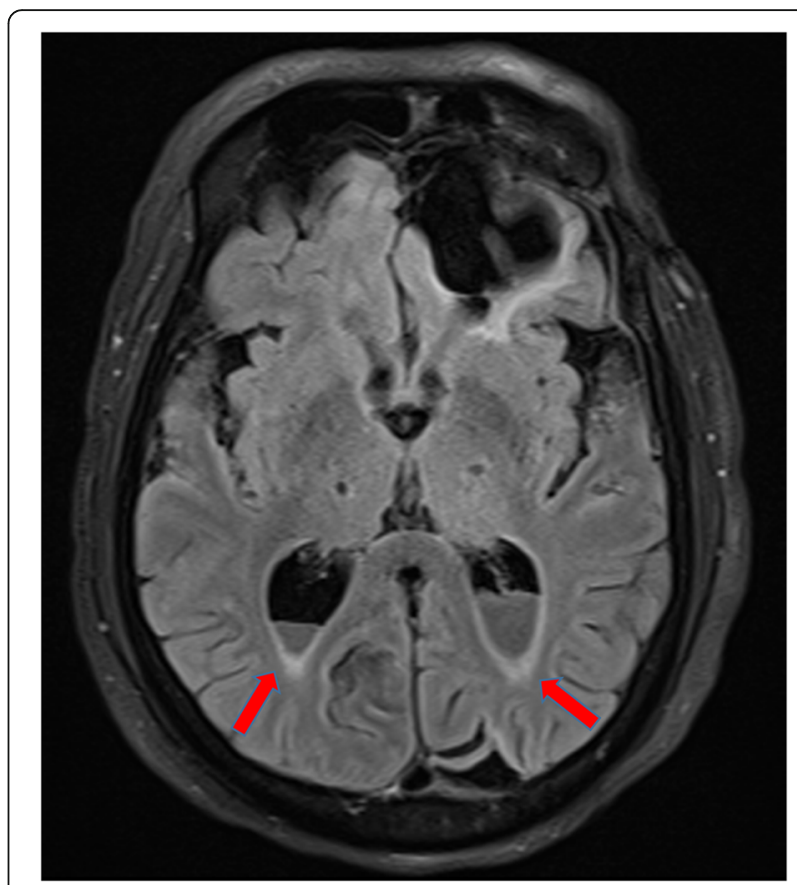

Fig. 5 Post-gadolinium T2 FLAIR showing subtle ventricular ependymal enhancement and exudates (arrow) in occipital horns

across the world [1]. Various risk factors have been identified for the same. Among them, alcoholism with cirrhosis, uncontrolled diabetes, disseminated strongyloidiasis, HIV, and chronic organ dysfunction are the most common [1]. Previously reported cases identified the primary focus of E. coli from the urinary tract or GI tract, and a rare case report has identified the source as a retropharyngeal abscess [4]. In our case, the patient underwent a neurosurgical procedure for CSF leak two decades ago. He neither had symptoms of a leak nor an evidence of CSF leak could be demonstrated during this admission. Hence, this meningitis cannot be labeled as postsurgical meningitis. Presence of subtle features of ventriculitis on imaging makes it further uncommon, especially when it is community-acquired. Preantibiotic era has shown presence of ventriculitis at the end of the first week in pathological examinations, but the current incidence is very low [5] and the literature was silent especially on community-acquired $E$. coli meningitis. At the same time, we could not find the primary source for $E$. coli in our case. This could be explained by the prior oral antibiotics taken at the time of onset of fever. Presence of filamentous gram-negative rods in microscopy also strengthens the prior antibiotic exposure [6]. We tried to look into other rare causes like strongyloidiasis and chronic organ insufficiency which were absent in our patient. In this patient, possible source of $E$. coli could be from the urinary tract as there was a renal calculus. 


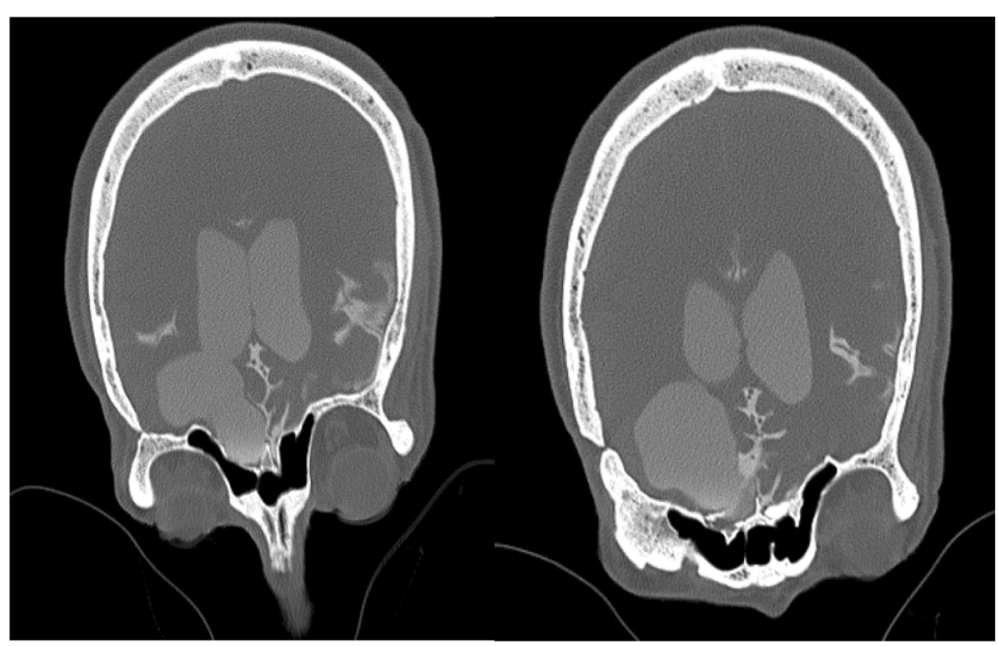

Fig. $6 \mathrm{CT}$ cisternography showing no contrast leak into paranasal sinuses

However, urine culture was negative. Ruling out all possible sources of $E$. coli, the infection might be communityacquired in our case.

Most commonly, wild type of E. coli causes meningitis up to $20 \%$ of cases [1]. Emergence of extended spectrum betalactamase (ESBL) production at community level in E. coli is a concern. Bichon et al. reported that up to 7\% of community-acquired infections with $E$. coli show ESBL production and $9 \%$ of $E$. coli show broad spectrum betalactamase production with resistance to penicillin group of antibiotics [1]. In our case report, it is a broad spectrum betalactamase producer with sensitivity to third-generation cephalosporins. While there are precise guidelines for the management of ventricularcatheter-related infections [5], we found neither recommendations nor expert advice regarding the optimal regimen or duration of the management of the treatment of primary bacterial ventriculitis. A 6- to 12-week duration of treatment, similar to recommendation for brain abscesses [7], was deemed essential in ventriculitis. However, given the severity of disease, a longer duration of antibiotics has to be given if the tolerance is acceptable.

Mortality with community-acquired $E$. coli meningitis is significant, and it ranges from 50 to $90 \%$, reaching to $85 \%$ in disseminated Strongyloidiasis and up to $100 \%$ in case of cirrhosis. Presence of multi-organ dysfunction worsens the chances of recovery. But in the recent review, Bichon et al. identified mortality as low as $47 \%$ with possible chances of recovery.

In conclusion, community-acquired $E$. coli meningitis with ventriculitis in adults is described as a rare entity and is likely to be underrated and under-recognized. Newer risk factors identified, like urinary tract infection, digestive tract disorders, disseminated Strongyloidiasis, have to be verified in all proven cases. Emergence of ESBL production in wild type of $E$. coli is a concern with over the counter availability of antibiotics. Finally, the prognosis of meningitis with ventriculitis is determined by the diagnosis, timeliness, and adequate duration of antibiotic therapy.

\section{Abbreviations}

CSF: Cerebrospinal fluid; CT: Computerized tomography; E. coli: Escherichia coli; ESBL: Extended spectrum betalactamase; FLAIR: Fluid-attenuated inversion recovery; GCS: Glasgow coma scale; GNB: Gram-negative bacilli; MRI: Magnetic resonance imaging; WBC: White blood cells

\section{Acknowledgement}

Sandeep Gajbe and Neelima Karri contributed in collecting the material.

\section{Authors' contributions}

RK has contributed to and conceptualized the case report apart from the clinical management of the patient and interpreted the data regarding meningitis. He had a thorough research of the literature and given the maximum contribution to the manuscript. He coordinated among the other departments in collecting the information. SKS has contributed in the literature review and contributed to the manuscript. He guided in interpreting the patient data regarding meningitis and given the fine tuning to the script. MA has contributed in collecting the microscopic pictures and analyzed the microbiological information. All authors have read and approved the final manuscript.

Ethics approval and consent to participate

Ethics approval is not applicable, and consent to participate has been taken from the subject.

\section{Consent for publication}

Consent has been taken from the subject.

\section{Competing interests}

The authors declare that they have no competing interests.

\section{Publisher's Note}

Springer Nature remains neutral with regard to jurisdictional claims in published maps and institutional affiliations. 


\section{Author details}

'Department of Critical Care Medicine, Yashoda Hospitals, Alexander Road, PIN: 500003, Secunderabad, Telangana, India. ${ }^{2}$ Department of Neurology, Yashoda Hospitals, Secunderabad, India. ${ }^{3}$ Department of Microbiology,

Yashoda Hospitals, Secunderabad, India.

Received: 27 June 2018 Accepted: 13 September 2018

Published online: 24 September 2018

\section{References}

1. Bichon A, Aubry C, Dubourg G, Drouet H, Lagier RD, Parola P, et al. Escherichia coli spontaneous community-acquired meningitis in adults: a case report and literature review. Int J Inf Dis. 2018:67:70-4.

2. Durand ML, Calderwood SB, Weber DJ, Miller SI, Southwick FS, Caviness VS Jr, et al. Acute bacterial meningitis in adults. A review of 493 episodes. N Engl J Med. 1993;328:21-8.

3. Pomar V, Benito N, López-Contreras J, Coll P, Gurguí M, Domingo P. Spontaneous gram-negative bacillary meningitis in adult patients: characteristics and outcome. BMC Infect Dis. 2013;13:451.

4. Kohlmann R, Nefedev A, Kaase M, Sören G, et al. Community-acquired adult Escherichia coli meningitis leading to diagnosis of unrecognized retropharyngeal abscess and cervical spondylodiscitis: a case report. BMC Infect Dis. 2015;15:567-72.

5. Tunkel AR, Hasbun R, Bhimraj A, Byers K, Kaplan SL, Scheld WM, et al. 2017 Infectious Diseases Society of America's clinical practice guidelines for health care associated Ventriculitis and meningitis. Clin Infect Dis. 2017;64: 701-6.

6. Kini AR, Peterson LC. Filamentous Forms of Escherichia coli in Cerebrospinal Fluid. Arch Pathol Lab Med 2001;125:841.

7. Brouwer MC, Tunkel AR, McKhann GM, van de Beek D. Brain abscess. N Engl J Med. 2014:371:447-56.

Ready to submit your research? Choose BMC and benefit from:

- fast, convenient online submission

- thorough peer review by experienced researchers in your field

- rapid publication on acceptance

- support for research data, including large and complex data types

- gold Open Access which fosters wider collaboration and increased citations

- maximum visibility for your research: over $100 \mathrm{M}$ website views per year

At $\mathrm{BMC}$, research is always in progress.

Learn more biomedcentral.com/submissions 